\title{
Generation and characterization of rabbit polyclonal antibodies against Vasohibin-2 for determination of its intracellular localization
}

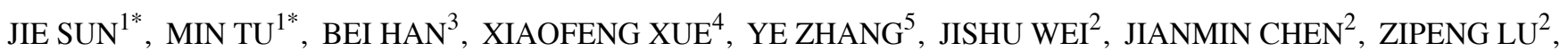 \\ YONG AN $^{1}$, BAOBAO CAI $^{1}$, NAN LV $^{1}$, KUIRONG JIANG ${ }^{2}$, YI MIAO ${ }^{1,2}$ and WENTAO GAO ${ }^{2 *}$ \\ ${ }^{1}$ Laboratory of General Surgery, ${ }^{2}$ Department of General Surgery, The First Affiliated Hospital of Nanjing Medical University; \\ ${ }^{3}$ Department of Endocrinology, Nanjing Children's Hospital Affiliated to Nanjing Medical University, Nanjing; \\ ${ }^{4}$ Department of General Surgery, The First Affiliated Hospital of Suzhou University, Suzhou; \\ ${ }^{5}$ Department of General Surgery, Wuxi People's Hospital, Wuxi, P.R. China
}

Received February 16, 2013; Accepted April 2, 2013

DOI: $10.3892 /$ ijo.2013.1919

\begin{abstract}
Vasohibin-2 was recently identified as an important pro-angiogenesis factor in solid tumor and intracellular localization of its variants is important for elucidating the downstream mechanism(s) of its effects. Currently there are no reported antibodies affordable for intracellular localization. The aim of this study was to generate and characterize polyclonal antibodies against Vasohibin-2 and to determine the intracellular localization of Vasohibin-2. In this study, two polypeptides were synthesized and one prokaryotic Vasohibin-2 recombinant protein was custom-made. New Zealand rabbits were immunized with the polypeptide mixture and prokaryotic recombinant protein, respectively. The purified antibodies from the antiserum were validated by ELISA, western blotting (WB), immunofluorescence (IF), immunohistochemistry (IHC) and immunoprecipitation (IP). In order to determine intracellular localization, the cytoplasmic and nuclear proteins of the human liver cancer cell line HepG2 were isolated for the detection of Vasohibin-2 by western blotting. Vasohibin-2 cDNA, coding for 311 and 355 amino acid residues, fused with or without a DDK/V5 tag at the c-terminus, respectively, was cloned into the Lv-CMV-EGFP vector. Lentiviruses were successfully packaged. Vasohibin-2-overexpressing HepG2VASH2 (355 amino acid residues) and HepG2-VASH2-V5 (311 amino acid residues fused with V5 tag at the c-terminus) human liver cancer cell lines were established. Approximately
\end{abstract}

Correspondence to: Professor Yi Miao or Professor Wentao Gao, Department of General Surgery, The First Affiliated Hospital of Nanjing Medical University, 300 GuangZhou Road, Nanjing 210029, P.R. China

E-mail: miaoyi@njmu.edu.cn

E-mail: gao11@hotmail.com

*Contributed equally

Key words: Vasohibin-2, polyclonal antibody, intracellular localization 1-2x10 $1{ }^{6}$ HepG2, HepG2-VASH2 and HepG2-VASH2-V5 cells were injected subcutaneously into the flanks of BALB/c nude mice. Xenograft tumors were harvested for immunohistochemistry. HepG2 cells were transiently transfected with the Lv-CMV-EGFP vectors containing Vasohibin-2 cDNA (coding for 311/355 amino acid residues with a DDK tag at the c-terminal), followed by anti-DDK immunofluorescence. The antibodies obtained were able to detect human VASH2 successfully as applied in western blotting, IF, IHC and IP. Results from IF, IHC and WB (post cytoplasmic/nuclear protein isolation) showed a quite different intracellular localization of VASH2 protein. The VASH2 (with 355 amino acid residues) was located in the cytoplasm while VASH2 (with 311 amino acid residues) was located in the nucleus. The former was found to be a relatively low abundance protein. We successfully generated three rabbit anti-human Vasohibin-2 polyclonal antibodies which can be used for western blotting, IF, IP and IHC. These antibodies will provide a convenient tool for further studies on Vasohibin-2. This is the first study to report differences in the intracellular localization of the VASH2 protein and, hence, a new research direction on the study of VASH2.

\section{Introduction}

Vasohibin-2 (VASH2) belongs to the Vasohibin family which is composed of Vasohibin-1 (VASH1) and VASH2. The human VASH2 gene, firstly identified by Shibuya et al, is reported to be located on chromosome 1q32.3 and composed of 355 amino acid residues (1-3). VASH2 is one novel gene homologous to VASH1 and the overall homology between them is $>50 \%$ at the amino acid level (1,3-5). VEGF is found to induce VASH1 expression in human umbilical vein endothelial cells (HUVECs) (6) similarly to fibroblast growth factor 2 (FGF-2) $(7,8)$. VASH1 was identified to be an intrinsic and specific feedback inhibitor playing an important role in activating ECs engaged in angiogenesis $(7,9,10)$, while exogenous VASH1 hindered sprouting angiogenesis by solid tumors $(11,12)$. In recent studies, it was found that, expression of VASH1 is 
wide-spread rather than confined to the ECs $(13,14)$. However, unlike VASH1, VASH2 has been found to promote angiogenesis and is identified as an extrinsic and VEGF-independent angiogenic factor $(1,15)$. In recent years, there is increasing research on the interrelation between VASH2 and carcinoma. VASH2 is highly expressed in carcinoma, and functions as a tumor growth promoter by angiogenesis $(15,16)$. The accurate mechanism needs to be further investigated. Currently, there are few VASH2 antibodies in the market which can be applied only in western blotting. The exact intracellular localization of VASH2 is also still unknown, which makes further research on VASH2 difficult.

In our study, we selected New Zealand rabbit as the animal for polyclonal antibody production because it has larger antibody repertoire possessing a higher specificity in recognizing conformational and modified apitopes than do mouse antibody (17-19). We immunized the rabbits separately with a mixture of two specific polypeptides coupled with keyhole limpet hemocyanin (KLH) and a custom-made prokaryotic recombinant part-length VASH2 protein. Antibodies purified from the antiserum were identified by western blotting, immunofluorescence (IF), immunohistochemisty (IHC) and immunoprecipitate (IP). In order to further investigate specific recognition capability of the antibodies, the VASH2 cDNA (encoding for 355 and 311 amino acid residues) was cloned into the Lv-CMV-EGFP vector, respectively. We also synthesized a eukaryotic recombinant VASH2 protein (311 amino acid residues). We found that, VASH2 proteins (355 and 311 amino acid residues) were successfully recognized by our prepared antibodies. IF, IHC and WB (post cytoplasmic/nuclear protein isolation) results showed clear differences in intracellular localization of VASH2 protein where 355 amino acid residues were found in the cytoplasm while 311 amino acid residues in the nucleus. From this study, we also found a new localization of VASH2 protein (311 amino acid residues) which may show us a new research direction. The preparation of anti-VASH2 polyclonal antibodies will provide a useful tool and facilitate further study on VASH2.

\section{Materials and methods}

Bioinformatics analysis and production of immunogens. Firstly, the sequence of VASH2 protein (355 amino acid residues) was analyzed by DNASTAR Protein 5.01 (Madison, WI, USA). Then we selected two sections of the VASH2 protein with high antigenicity, low hydrophobicity and favorable surface exposure to synthesize polypeptides as immunogens. The polypeptides were synthesized by GL Biochem (Shanghai, China). In addition, a prokaryotic recombinant part-length VASH2 protein was designed and produced by Abmart (Shanghai, China).

Preparation and purification of antibodies. All of the experimental procedures were approved by the Animal Care and Use Subcommittee at Nanjing Medical University. Eight New Zealand rabbits (Jiangsu Agricultural Academy of Science, Nanjing, China), all males, weighing between 2.0 and $2.5 \mathrm{~kg}$, were used and allowed free access to food and water. Peripheral blood was extracted before immunization and serum used as antibody (1:200) to perform western blotting (HepG2 cell
Table I. Immunogens for preparation of anti-VASH2 polyclonal antibodies.

\begin{tabular}{lllc}
\hline Name & Location & Amino acid sequence & $\begin{array}{c}\text { Polypeptide } \\
\text { length }\end{array}$ \\
\hline S1 & aa211-323 & RRAELMD-RQASPP & 113aa \\
S2 & aa317-327 & RRQASPPRRG & 11 aa \\
S3 & aa300-311 & AHSPTQVRSRGK & 12 aa \\
\hline
\end{tabular}

The polypeptides and recombinant protein purities were 95 and $74 \%$, respectively.

lysate). After screening by western blotting, we obtained two rabbits with no obvious band. These selected two rabbits were immunized subcutaneously with $100 \mu \mathrm{g}$ prokaryotic recombinant part-length VASH2 protein. Another two unscreened rabbits were immunized subcutaneously with a mixture of two polypeptides (each peptide $100 \mu \mathrm{g}$ ). Immune solution preparation and procedure were performed as described (20). We harvested the antiserum from the rabbit carotid artery 8 weeks later after 4-5 subcutaneous immunizations (21). The antiserum titer was determined by indirect ELISA (22-24). The antisera were purified by immuno-affinity purification or protein-G column purification by Shanghai GL Biochem when the titer reached 1:25,000.

Cell cultures and tissue sections. Human liver cancer cell line HepG2 was a gift of Professor Beicheng Sun (Department of General Surgery of The First Affiliated Hospital of Nanjing Medical University, Nanjing, China). The cell line was cultured in DMEM (Wisent, Canada) provided with $10 \%$ fetal bovine serum (Wisent, Canada) at $37^{\circ} \mathrm{C}$ with $5 \% \mathrm{CO}_{2}$. Human liver cancer and adjacent normal tissues were obtained from patients from the First Affiliated Hospital of Nanjing Medical University. The patients were informed and their detailed information and written consents were attained.

Plasmid construction, transient transfection and lentivirus packaging. The plasmid highly expressed VASH2 (355 amino acid residues) fused with DDK tag at the c-terminal was constructed as described (15). Full-length VASH2 cDNA (encoding for 311 amino acid residues) bought from Origene (USA) was fused with DDK or V5 tag at the c-terminal and cloned into the Lv-CMV-EGFP vector. All plasmids were verified by sequencing (Invitrogen, Shanghai, China). Lipofectamine 2000 (Invitrogen) was used as transient transfection reagent according to the manufacturer's instructions. Lentivirus packaging method was the same as described (15). The primer pair for the VASH2 plasmid (311 amino acid residues) was as follows: forward, 5'-CGGCTAGCCCCACCA TGACCGGCTC-3' and reverse, 5'-AACTGCAGCTACTTATC GTCGTCATCCTTGTAATCAATTCGGATTTGATAGCCC ACTT-3' (DDK-tag), 5'-AACTGCAGCTACGTAGAATCGA GACCGAGGAGAGGGTTAGGGATAGGCTTACCAATTC GGATTTGATAGCCCACTT-3' (V5-tag). After transfection with the lentivirus, the VASH2 highly expressed cells were tested by western blotting and qRT-PCR. 
Table II. Detailed summary of the prepared polyclonal antibodies against VASH2 and their applications.

\begin{tabular}{lllcc}
\hline Name & Serum titer & \multicolumn{1}{c}{ Purification methods } & Concentration (mg/ml) & Applications \\
\hline S1 & $1.0 \times 10^{5}$ & Protein-G column & 2.00 & WB, IF \\
S2 & $5.0 \times 10^{4}$ & Immunoaffinity purification & 1.60 & WB, IP, IF, IHC \\
S3 & $2.5 \times 10^{4}$ & Immunoaffinity purification & 0.14 & WB, IF \\
\hline
\end{tabular}

Table III. Indirect sandwich ELISA analysis results of anti-VASH2 antibodies.

\begin{tabular}{lcccccccrrr}
\hline Antibody ID & $2 \mathrm{k}$ & $4 \mathrm{k}$ & $8 \mathrm{k}$ & $16 \mathrm{k}$ & $32 \mathrm{k}$ & $64 \mathrm{k}$ & $128 \mathrm{k}$ & $256 \mathrm{k}$ & $\mathrm{PC}$ & $\mathrm{NC}$ \\
\hline S1 & 2.137 & 1.426 & 0.824 & 0.464 & 0.370 & 0.274 & 0.194 & 0.122 & 2.475 & 0.131 \\
S2 & 2.118 & 1.284 & 0.778 & 0.585 & 0.414 & 0.276 & 0.177 & 0.155 & 2.472 & 0.121 \\
S3 & 2.167 & 1.438 & 0.856 & 0.519 & 0.329 & 0.234 & 0.176 & 0.152 & 2.516 & 0.119
\end{tabular}

Positive control (PC), polyclonal antiserum without purification; negative control (NC), IgG purified from normal rabbit sera. The antibody titer is the dilution value corresponding to OD450 value which is twice greater than $\mathrm{NC}$ and $>0.25$.

Mouse xenograft models. Female, 3-4-week-old BALB/c nude mice were obtained from Nanjing Medical University. Aliquots of HepG2, HepG2-VASH2 (355 amino acid residues) and HepG2-VASH2-v5 (311 amino acid residues fused with V5 tag at the C-terminal $)$ cells $\left(0.1 \mathrm{ml}, 1-2 \times 10^{6}\right.$ cells $)$ were injected into flanks of mice. Four to six weeks later, xenograft tumors were harvested and fixed in $4 \%$ neutral formalin.

Recombinant protein, nuclear extraction analysis and western blotting. The VASH2 recombinant protein (311 amino acid residues) was purchased from Origene (USA). The cytoplasmic and nuclear samples were isolated using a Nuclear Extraction kit 2900 (Millipore, MA, USA). The experimental procedures were the same as in the supplier's manual. Cell lysates were prepared by extracting protein with radio immunoprecipitation assay buffer. PVDF membranes (Millipore) were blocked in $5 \%$ skim milk and incubated overnight at $4{ }^{\circ} \mathrm{C}$ with the three rabbit antibodies prepared at a dilution of 1:3,000 (S1), 1:3000 (S2) and 1:200 (S3), respectively. A mouse anti-human VASH2 antibody, as a verified antibody $(2,15)$, was a gift from Professor Y. Sato (Department of Vascular Biology, Institute of Development, Aging and Cancer, Tohoku University, Sendai, Japan). Both the anti-GAPDH and secondary antibodies were purchased from Beyotime. The rabbit anti-human $\mathrm{H} 3$ antibody was purchased from Cell Signaling (USA) while the rabbit anti-V5 and mouse anti-human E-cadherin antibodies were from Abcam (USA).

Immunoprecipitation (IP). Pierce Co-IP kit (Thermo, USA) was used for immunoprecipitation analysis according to the supplier's manual. The prepared antibodies were coupled with resin (20 $\mu \mathrm{g}$ antibody $/ 25 \mu \mathrm{l}$ resin) and then incubated in $3.0 \mathrm{ml}$ HepG 2 cell lysate $\left(\sim 3 \times 10^{7}\right.$ cells) with gentle mixing overnight at $4^{\circ} \mathrm{C}$. Eluted protein samples were added with SDS-PAGE sample buffer and boiled at $95^{\circ} \mathrm{C}$ for $5 \mathrm{~min}$. The normal rabbit $\mathrm{IgG}$ (Millipore) was used as negative control.
Immunofluorescence. HepG2 cell line was arrayed in a 24-well plate (Corning Inc., USA), and IF was performed as described $(25,26)$. Three polyclonal antibodies were diluted as follows: S1, 1:200, S2, 1:400, S3, 1:50. Mouse-anti-DDK antibody was from Abmart. Second antibody: goat anti-rabbit IgG-dylight 593 (Invitrogen, USA). Nucleus dye, $10 \mu \mathrm{g} / \mathrm{ml}$ DAPI (Sigma, St. Louis, MO, USA) diluted in PBS. Finally, cells were observed under a fluorescence microscope (Olympus, Japan).

Immunohistochemistry. The immunohistochemical staining procedure was performed as described $(27,28)$. Histological sections $(5 \mu \mathrm{m})$ were analyzed by immunohistochemistry using the prepared antibodies. The test dilution of antibodies was 1:50-1:1,000.

\section{Results}

Based on the DNASTAR software and comprehensive analysis, we selected two sections of VASH2 as polypeptides for synthesizing. The polypeptides had low homology to rabbit and human protein sequences. The details of the two polypeptides and one prokaryotic recombinant part-length VASH2 protein are shown in Table I. We selected two rabbits with no obvious band after screening by western blotting using the preimmune serum as antibody (data not shown).

The antisera were purified using Shanghai GL Biochem kit and details of the three antibodies are shown in Table II. To identify the specificity of our antibodies, their reactions (S1-S3) with antigens were tested by indirect sandwich ELISA. Results indicate that the optimal concentration for the recombinant antigens was $5 \mu \mathrm{g} / \mathrm{ml}$, the titer for $\mathrm{S} 1, \mathrm{~S} 2$ was $1: 64,000$ while for S3 was 1:32,000 (Table III).

In order to identify the specificity of the antibodies against VASH2, western blotting was performed. In HepG2, we observed a single band at $34 \mathrm{kDa}$, while two bands at 41 and $34 \mathrm{kDa}$ were detected in HepG2-VASH2 (355 amino acid residues) (Fig. 1A). To further demonstrate recognition capability 


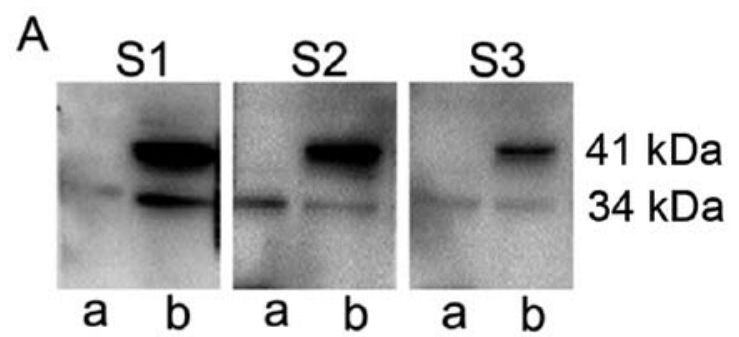

C
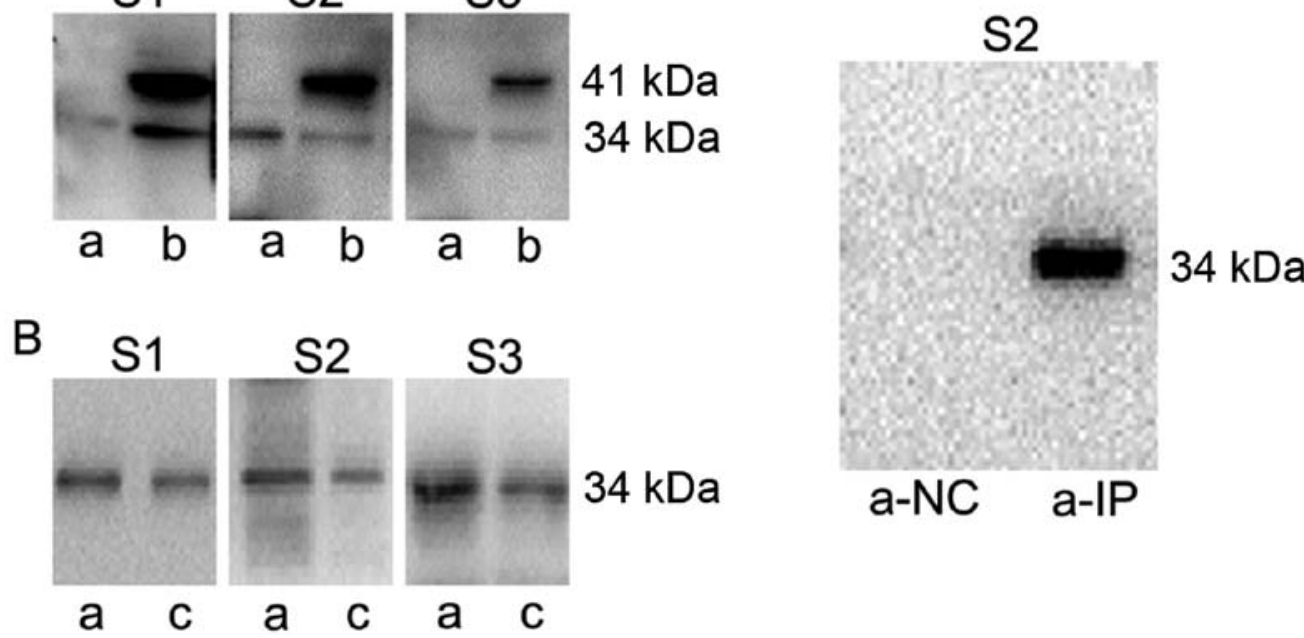

Figure 1. Western blot and IP analyses of the three polyclonal antibodies. a, HepG2; b, HepG2-VASH2 (355 amino acid residues); c, the recombinant protein of VASH2 (311 amino acid residues). (A) A single band of the relative molecular weight $34 \mathrm{kDa}$ was observed in HepG2 while two bands at 41 and $34 \mathrm{kDa}$ were detected in HepG2-VASH2 (355 amino acid residues). (B) A single band at $34 \mathrm{kDa}$ was detected in HepG2 and VASH2 recombinant protein with each (S1-3) antibody. (C) Only the S2 antibody performed successfully, with a clear band at $34 \mathrm{kDa}$ as observed in the figure (NC, normal rabbit IgG).

to $34 \mathrm{kDa}$ VASH2 protein, the VASH2 recombinant protein (311 amino acid residues) purchased from Origene was tested (Fig. 1B). The results indicate that all the prepared polyclonal antibodies have high specificity to VASH2 protein (311 amino acid residues).

Only the S2 antibody performed successfully in IP analysis. No band in a-NC (normal rabbit $\mathrm{IgG}$ ), while a clear band at $34 \mathrm{kDa}$ is observed in a-IP (Fig. 1C).

IF analysis was carried out using the HepG2 cell line (Fig. 2). The results demonstrate that the three antibodies can be used for IF. Moreover, we found that human VASH2 protein was mainly localized in the nucleus and only slight in the cytoplasm.

Human liver cancer, adjacent normal tissues and the harvested xenograft tumor tissues were tested by IHC. The results revealed that only $\mathrm{S} 2$ possessed the specificity and sensitivity to recognize VASH2 protein in IHC (Fig. 3). Fig. 3 shows that the VASH2 protein is mainly localized in the nucleus. In the HepG2-VASH2 (355 amino acid residues), we observed that the cytoplasm was deeply stained compared to HepG2, meaning that the overexpressed VASH2 (355 amino acid residues) was mainly in the cytoplasm. In the human liver cancer adjacent normal tissue, we observed that the VASH2 protein was mainly localized in the nucleus while only slight in the cytoplasm. A question arose as to whether the two VASH 2 proteins (355 amino acid residues and 311 amino acid residues) had different intracellular localizations.

In order to further confirm the intracellular localization of the two VASH2 proteins, the cytoplasmic and nuclear cellular extracts were prepared and then analyzed by western blotting. We tested localization effects of nuclear/cytoplasmic proteins using E-cadherin, H3 and GAPDH (Fig. 4A). The E-cadherin band was observed only in the cytoplasmic extracts while the $\mathrm{H} 3$ band was in the nucleus. The GAPDH band was observed mainly in the cytoplasmic extracts while only slightly in the nuclear extracts. These results demon- strate that the nuclear and cytoplasmic proteins used to show localization effects were successfully isolated. Using the verified mouse anti-human VASH2 antibody $(11,15)$ and the prepared rabbit polyclonal antibodies (S2, S3), we observed that, exogenous VASH2 (41 kDa, 355 amino acid residues) was mainly located in the cytoplasm while endogenous VASH2 (34 kDa, 311 amino acid residues) was located in the nucleus (Fig. 4A). A slight band at $41 \mathrm{kDa}$ in the HepG2-VASH2 nucleus (Fig. 4A) was considered to be a result of negligible contamination of cytoplasmic protein. To further verify the nuclear localization of VASH2, IF analysis using the anti-DDK antibody was performed for the HepG2-VASH2 (transient overexpressed c-terminal DDK-tagged 311 and 355 amino acid residues VASH2). The HepG2-VASH2 (311 amino acid residues) nucleus was stained red while the cytoplasm was red in the HepG2-VASH2 (355 amino acid residues) under the fluorescence microscope (Fig. 4B). IHC results from the mouse xenograft (HepG2-VASH2-V5, 311 amino acid residues) show that V5 was localized both in the cytoplasm and nucleus (Fig. 4C). In Figs. 1A and 4A, no band at $41 \mathrm{kDa}$ was observed in the HepG2 lysate while in the HepG2 IF (Fig. 2) and HepG2 IHC (Fig. 3a), slight stain in the cytoplasm was observed. These results indicate that the endogenous VASH2 protein exists in the cytoplasm. When double dose of HepG2 cytoplasmic lysates were loaded as western blotting samples, a slight band at $41 \mathrm{kDa}$ in the HepG2 cytoplasm was observed (Fig. 5).

\section{Discussion}

VASH2, a novel gene homologous to VASH1, is considered as an angiogenesis promoter (2). Recently, it has been found that VASH2 plays an important role in carcinoma angiogenesis and malignant transformation (15). VASH2 is also reported to be expressed in gastric cancer cells (4). The available data demonstrate that VASH2 is associated with carcinoma and 

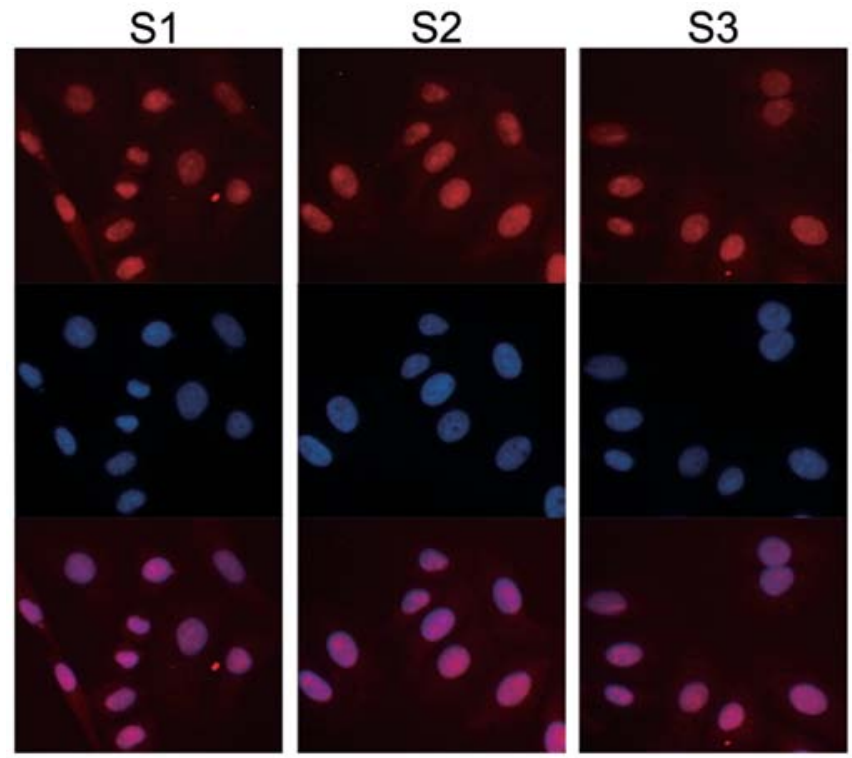

VASH2

HepG2

Figure 2. Immunofluorescence staining of HepG2, magnification, x400. The first row shows distribution patterns of anti-VASH2 antibodies (S1-S3) in HepG2. The second row shows the nuclei staining with DAPI for HepG2. The last row shows merged first and second rows.

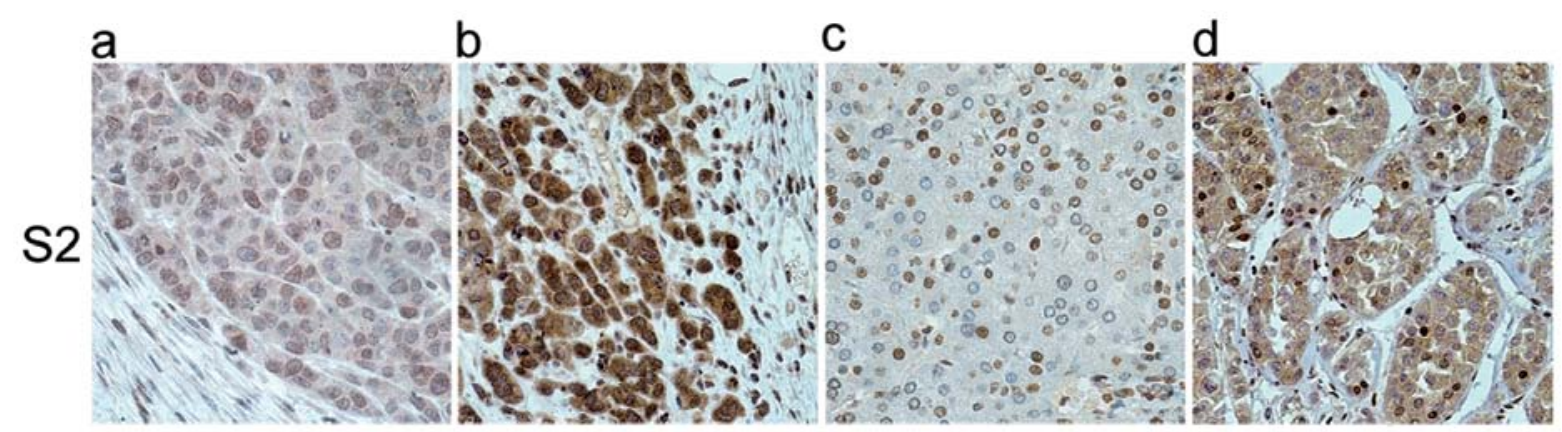

Figure 3. IHC analysis of VASH2 in HepG2/HepG2-VASH2 (355 amino acid residues) xenograft tumor tissues (a and b), human liver adjacent normal tissue (c), and human liver cancer tissue (d) magnification, x200. In (a) we observed that the VASH2 protein was mainly localized in the nuclei; (b) the cytoplasm was deeply stained; (c) the VASH2 protein was mainly localized in the nuclei while only slightly in the cytoplasm; (d) both the cytoplasm and nucleus were stained.

A

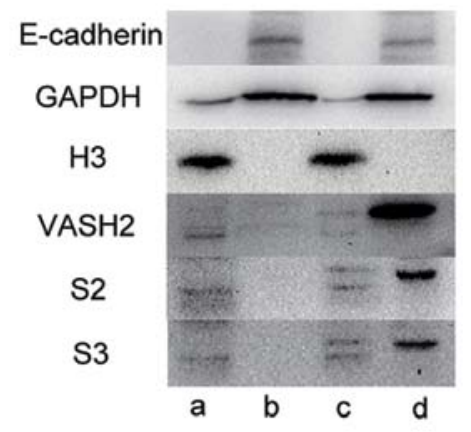

B

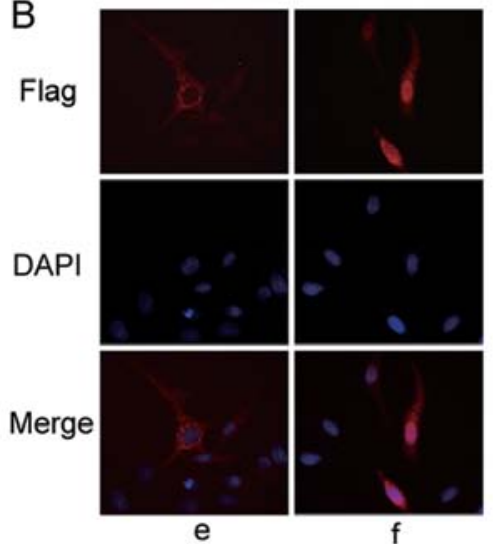

$\mathrm{C}$

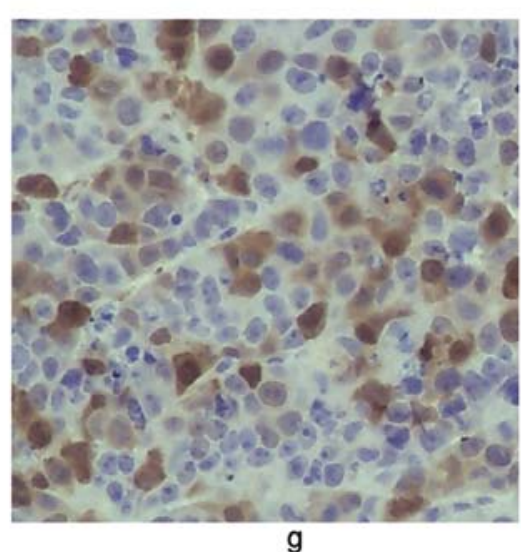

Figure 4. Intracellular localization of the VASH2 protein. a, HepG2-nucleus; b, HepG2-cytoplasm; c, HepG2-VASH2-nucleus (355 amino acid residues); d, HepG2-VASH2-cytoplasm (355 amino acid residues); e, HepG2-VASH2-Flag (355 amino acid residues) magnification, x400; f, HepG2-VASH2-Flag (311 amino acid residues) magnification, x400; g, mouse xenograft tumor tissue (HepG2-VASH2-V5, 311 amino acid residues) magnification, x200. (A) The cytoplasmic and nuclear cellular extracts prepared and analyzed by western blotting. (B) IF analysis with the anti-DDK antibody performed in HepG2-VASH2 (transient overexpressed c-terminal DDK-tagged 311 and 355 amino acid residues VASH2). (C) IHC analysis performed in the mouse xenograft tumor tissue (HepG2-VASH2-V5, 311 amino acid residues). 


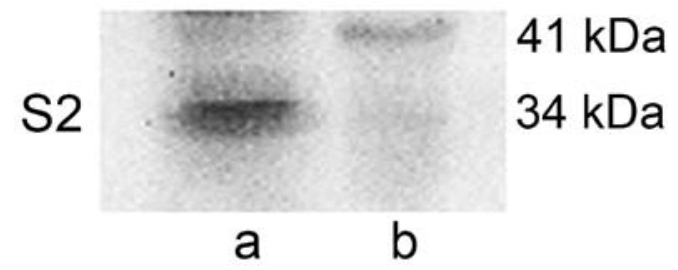

Figure 5. Western blot analysis of the VASH2 protein intracellular localization in HepG2. a, HepG2-nucleus; b, HepG2-cytoplasm. The double dose of HepG2 cytoplasmic lysates was analyzed by Western blotting. A clear band at $34 \mathrm{kDa}$ was observed in the HepG2 nucleus (a) while a slight band at $41 \mathrm{kDa}$ was observed in the HepG2 cytoplasm (b).

angiogenesis. Although the accurate mechanism involving VASH2 and angiogenesis in carcinoma is unknown, VASH2 investigation may provide a novel target for tumor therapy. We have been studying VASH2 function and molecular mechanisms for several years. However, the supply of VASH2 antibodies in the market is very limited (available for western blotting only). This shortage has sometimes hindered further research on VASH2. Hence, effective VASH2 antibodies should be exploited for future research use. Herein, we have prepared and evaluated VASH2 polyclonal antibodies by western blotting, IF, IHC and IP. The detailed applications of these three antibodies are summarized in Table II. All the applications listed were achieved successfully in this study.

Formerly, VASH2 was reported as a secreted protein $(1,2,16)$. We analyzed both the 311 and 355 amino acid residues of VASH2 protein sequence using the protein subcellular localization predicting software Hum-mPLoc 2.0 (http:// www.csbio.sjtu.edu.cn/bioinf/hum-multi-2/). The prediction result showed extracellular localization for both. This result was not consistent with our present data. Then, we used the Nakai Server software (http://psort.hgc.jp/form.html) and the result showed nuclear localization while the peptide sequence 'RRRQASPPRRLGRREKS' was predicted as nuclear location signal. Our present data showed that, full length VASH2 composed of 355 amino acid residues was located in the cytoplasm while the 311 amino acid residues VASH2 in the nucleus. This was verified by western blot analysis of cytoplasmic/nuclear cellular extracts using the verified mouse anti-human VASH2 antibody $(11,15)$ and the prepared rabbit polyclonal antibodies (S2, S3). From these results we observed that, exogenous VASH2 (41 kDa, 355 amino acid residues) was mainly located in the cytoplasm while endogenous VASH2 (34 kDa, 311 amino acid residues) was mainly located in the nucleus (Fig. 4A). Results from this study also indicate that endogenous VASH2 protein also exists in the cytoplasm. This was observed in the HepG2 IF (Fig. 2) and HepG2 IHC (Fig. 3a) where slight dyeing in the cytoplasm was observed. This was confirmed when double doses of HepG2 cytoplasmic lysates was analyzed by western blotting, giving a slight band at $41 \mathrm{kDa}$ in the HepG2 cytoplasm (Fig. 5). We thus also conclude that, both, the 311 and 355 amino acid residues of VASH2 protein have intracellular expression, but the latter is of low abundance. In human liver cancer tissue IHC (Fig. 3d), we observed that both the cytoplasm and nucleus were stained. Compared to human liver cancer adjacent tissue, the cytoplasm VASH2 protein was highly expressed. Because of inadequate number of cases, the result needs to be further demonstrated by expanding the number of cases.

VASH2 is closely related with carcinoma, but the specific downstream signaling pathway has not been found so far. In the past, VASH2 was considered as a single cytoplasm localized protein. Here, our study confirms that the different VASH2 protein isoforms have different intracellular localization, thus VASH2 proteins need to be divided into two types: karyo and cytoplasmic type.

In conclusion, we have successfully generated three rabbit anti-human VASH2 polyclonal antibodies which can be applied for western blotting, IF, IP and IHC. We also confirmed that the VASH2 protein has different intracellular localizations, 355 amino acid residues being mostly in the cytoplasm while 311 amino acid residues in the nucleus. The results from this study show us a new direction for further study on VASH2.

\section{Acknowledgements}

This study was supported by grants from NSFC (nos. 81172267 , 81170336 and 30972912) and the translational research of early diagnosis and comprehensive treatment in pancreatic cancer (201202007).

\section{References}

1. Kimura H, Miyashita H, Suzuki Y, Kobayashi M, Watanabe K, Sonoda H, Ohta H, Fujiwara T, Shimosegawa T and Sato Y: Distinctive localization and opposed roles of vasohibin-1 and vasohibin-2 in the regulation of angiogenesis. Blood 113: 4810-4818, 2009.

2. Shibuya T, Watanabe K, Yamashita H, Shimizu K, Miyashita H, Abe M, Moriya T, Ohta H, Sonoda H, Shimosegawa T, Tabayashi K and Sato Y: Isolation and characterization of vasohibin-2 as a homologue of VEGF-inducible endotheliumderived angiogenesis inhibitor vasohibin. Arterioscler Thromb Vasc Biol 26: 1051-1057, 2006.

3. Sato Y and Sonoda H: The vasohibin family: a negative regulatory system of angiogenesis genetically programmed in endothelial cells. Arterioscler Thromb Vasc Biol 27: 37-41, 2007.

4. Shen Z, Kauttu T, Seppänen H, Vainionpää S, Ye Y, Wang S, Mustonen $\mathrm{H}$ and Puolakkainen P: Vasohibin-1 and vasohibin-2 expression in gastric cancer cells and TAMs. Med Oncol 29: 2718-2726, 2012.

5. Sato Y: The vasohibin family: novel regulators of angiogenesis. Vascul Pharmacol 56: 262-266, 2012.

6. Abe M and Sato Y: cDNA microarray analysis of the gene expression profile of VEGF-activated human umbilical vein endothelial cells. Angiogenesis 4: 289-298, 2001.

7. Watanabe K, Hasegawa Y, Yamashita H, Shimizu K, Ding Y, Abe M, Ohta H, Imagawa K, Hojo K, Maki H, Sonoda H and Sato Y: Vasohibin as an endothelium-derived negative feedback regulator of angiogenesis. J Clin Invest 114: 898-907, 2004.

8. Shimizu K, Watanabe K, Yamashita H, Abe M, Yoshimatsu H, Ohta $\mathrm{H}$, Sonoda $\mathrm{H}$ and Sato Y: Gene regulation of a novel angiogenesis inhibitor, vasohibin, in endothelial cells. Biochem Biophys Res Commun 327: 700-706, 2005.

9. Nasu T, Maeshima Y, Kinomura M, Hirokoshi-Kawahara K, Tanabe K, Sugiyama H, Sonoda H, Sato Y and Makino H: Vasohibin-1, a negative feedback regulator of angiogenesis, ameliorates renal alterations in a mouse model of diabetic nephropathy. Diabetes 58: 2365-2375, 2009.

10. Shirasuna K, Kobayashi A, Nitta A, Nibuno S, Sasahara K, Shimizu T, Bollwein H and Miyamoto A: Possible action of vasohibin-1 as an inhibitor in the regulation of vascularization of the bovine corpus luteum. Reproduction 143: 491-500, 2012.

11. Tamaki K, Moriya T, Sato Y, Ishida T, Maruo Y, Yoshinaga K, Ohuchi N and Sasano H: Vasohibin-1 in human breast carcinoma: a potential negative feedback regulator of angiogenesis. Cancer Sci 100: 88-94, 2009. 
12. Yoshinaga $K$, Ito $K$, Moriya $T$, Nagase $S$, Takano $T$, Niikura $H$, Sasano H, Yaegashi N and Sato Y: Roles of intrinsic angiogenesis inhibitor, vasohibin, in cervical carcinomas. Cancer Sci 102: 446-451, 2011

13. Nimmagadda S, Geetha-Loganathan P, Pröls F, Scaal M, Christ B and Huang R: Expression pattern of Vasohibin during chick development. Dev Dyn 236: 1358-1362, 2007.

14. Naito H, Kidoya H, Sato Y and Takakura N: Induction and expression of anti-angiogenic vasohibins in the hematopoietic stem/progenitor cell population. J Biochem 145: 653-659, 2009.

15. Xue X, Gao W, Sun B, Xu Y, Han B, Wang F, Zhang Y, Sun J, Wei J, Lu Z, Zhu Y, Sato Y, Sekido Y, Miao Y and Kondo Y: Vasohibin 2 is transcriptionally activated and promotes angiogenesis in hepatocellular carcinoma. Oncogene: May 21, 2012 (Epub ahead of print). doi: 10.1038/onc.2012.177.

16. Takahashi Y, Koyanagi T, Suzuki Y, Saga Y, Kanomata N, Moriya T, Suzuki M and Sato Y: Vasohibin-2 expressed in human serous ovarian adenocarcinoma accelerates tumor growth by promoting angiogenesis. Mol Cancer Res 10: 1135-1146, 2012.

17. Huang Y, Gu B, Wu R, Zhang J, Li Y and Zhang M: Development of a rabbit monoclonal antibody group against Smads and immunocytochemical study of human and mouse embryonic stem cells. Hybridoma (Larchmt) 26: 387-391, 2007.

18. Spieker-Polet H, Sethupathi P, Yam PC and Knight KL: Rabbit monoclonal antibodies: generating a fusion partner to produce rabbit-rabbit hybridomas. Proc Natl Acad Sci USA 92: 9348-9352, 1995.

19. Tan Z, Zhang J, Su Z, Gu B, Jiang X, Luo J, Ji H, Wang G, Tao B, Zhao X, Chen L, Yu G, Zhu W and Zhang M: Production of rabbit monoclonal antibodies against mouse embryonic stem cells and identification of pluripotency-associated surface antigens. J Immunol Methods 365: 149-157, 2011.
20. Saradhi M, Krishna B, Mukhopadhyay G and Tyagi RK: Purification of full-length human pregnane and xenobiotic receptor: polyclonal antibody preparation for immunological characterization. Cell Res 15: 785-795, 2005.

21. Chen TF, Zhang YL, Xu WL, Li ZQ, Hou B, Wang CL, Fan M, Qian LJ, Zhou RP and Zhang CG: Prokaryotic expression, polyclonal antibody preparation, and sub-cellular localization analysis of $\mathrm{Na}^{+}, \mathrm{K}^{+}$-ATPase beta2 subunit. Protein Expr Purif 37: 47-52, 2004.

22. Crowther JR: ELISA. Theory and practice. Methods Mol Biol 42: 1-218, 1995

23. Crowther JR: The ELISA guidebook. Methods Mol Biol 149: III-IV, 1-413, 2000.

24. Tsumuraya T, Takeuchi K, Yamashita S, Fujii I and Hirama M: Development of a monoclonal antibody against the left wing of ciguatoxin CTX1B: thiol strategy and detection using a sandwich ELISA. Toxicon 60: 348-357, 2012.

25. Tang S, Wang Y, Zhang D, Gao Y, Ma Y, Yin B, Sun J, Liu J and Zhang Y: Reprogramming donor cells with oocyte extracts improves in vitro development of nuclear transfer embryos. Anim Reprod Sci 115: 1-9, 2009.

26. Lin J, Lin X, Yang GH, Wang Y, Peng BW and Lin JY: Toxoplasma gondii: expression of GRA1 gene in endoplasmic reticulum promotes both growth and adherence and modulates intracellular calcium release in macrophages. Exp Parasitol 125: $165-171,2010$.

27. Tan Z, Zhang J, Su Z, Gu B, Jiang X, Luo J, Ji H, Wang G, Tao B, Zhao X, Chen L, Yu G, Zhu W and Zhang M: Production of rabbit monoclonal antibodies against mouse embryonic stem cells and identification of pluripotency-associated surface antigens. J Immunol Methods 365: 149-157, 2011.

28. Hama $\mathrm{Y}$, Chano $\mathrm{T}$, Inui $\mathrm{T}$, Matsumoto $\mathrm{K}$ and Okabe $\mathrm{H}$ : Preparation of mouse monoclonal antibody for RB1CC1 and its clinical application. PLoS One 7: e32052, 2012. 